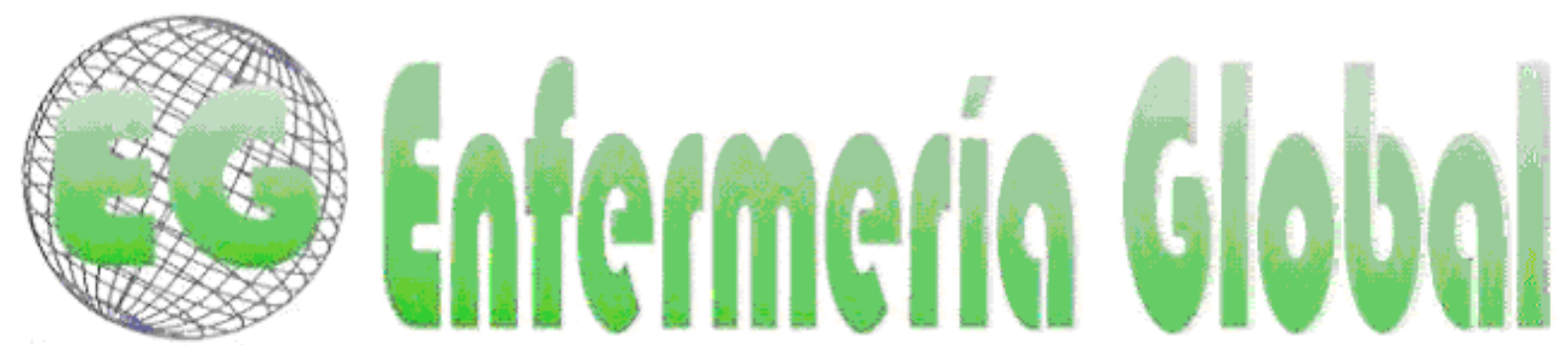

\title{
REVISIONES
}

\section{La relación de bioseguridad con el costo-efectividad en las hospitalizaciones: nexos con la formación continua}

A relação da biossegurança com o custo-efetividade nas hospitalizações: nexos com a educação permanente

The relation of biosafety at the cost-effectiveness in hospitalizations: nexus with the permanent instruction

\section{*Lopes Joaquim, Fabiana ${ }^{* *}$ Cavalcanti Valente, Geilsa Soraia}

\begin{abstract}
*Enfermera licenciada por la EEAAC / UFF. Especialista en Control de Infección en Asistencia a la Salud (EEAAC/UFF). E-mail: fabykim_enf@yahoo.com.br ${ }^{* *}$ Doctora en Enfermería (EEAN / UFRJ), Profesora Adjunta del Departamento de Fundamentos de Enfermería y Administración de la EEAAC /UFF. Rio de Janeiro, Brasil..
\end{abstract}

Palabras clave: Análisis Costo-Beneficio; capacitación; educación en enfermería; exposición a agentes biológicos.

Palavras-chave: Análise custo-benefício; capacitação; educação em enfermagem; exposição a agentes biológicos

Keywords: Cost-Benefit Analysis; training; education; nursing; exposure to biological agents

\section{RESUMEN}

Objetivos: Determinar si la bioseguridad puede ayudar a reducir el costo-efectividad de las hospitalizaciones, conocer el grado de impacto de la educación permanente en materia de bioseguridad para reducir los costos relacionados con la hospitalización y demostrar los efectos de la bioseguridad en la reducción de costos hospitalario basándose en la literatura existente.

Método: Estudio descriptivo exploratorio cualitativo, mediante la revisión sistemática de la literatura sobre la base de Enfermería de la base de datos (BDENF), América Latina y el Caribe en Ciencias de la Salud (LILACS) y Scientific Electronic Library Online (SCIELO).

Resultados: Se identificó la formación continua como un agente contribuyente a la concienciación de las enfermeras acerca de la seguridad necesaria en la profesión.

Conclusión: La bioseguridad asociada a la formación continua contribuye a la reducción del costorentabilidad 


\section{RESUMO}

Objetivos: Identificar se a biossegurança pode contribuir para reduzir o custo-efetividade nas hospitalizações; conhecer o grau de impacto da educação permanente para a biossegurança na redução dos custos relacionados à hospitalização e demonstrar os efeitos da biossegurança sobre a redução dos custos hospitalares com base na literatura existente.

Método: Estudo exploratório descritivo, qualitativo, através de revisão sistemática de literatura nas bases Banco de Dados da Enfermagem (BDENF), Literatura Latino-Americana e do Caribe em Ciências da Saúde (LILACS) e Scientific Electronic Library Online (SCIELO).

Resultados: Identificou-se a educação permanente como agente contribuinte na conscientização dos profissionais de enfermagem sobre a segurança necessária ao exercício profissional.

Conclusão: A biossegurança associada à educação permanente contribui para a redução do custoefetividade.

\section{ABSTRACT}

Aim: To identify whether the biosecurity can help to reduce the cost-effectiveness in hospitalizations; to know the degree of impact of permanent education for biosecurity in reducing costs related to hospitalization and to demonstrate the effects of biosecurity on reducing costs hospital-based literature.

Method: It is a descriptive exploratory study, qualitative, through systematic review of literature on the basis of Nursing Database (BDENF), Latin American and Caribbean Center on Health Sciences (LILACS) and Scientific Electronic Library Online (SCIELO).

Results: It was identified permanent education as an agent in contributing awareness of nurses about the security needed in the profession.

Conclusion: The biosafety related to permanent education contributes to the reduction of the costeffectiveness.

\section{INTRODUCCIÓN}

A lo largo de la trayectoria académica, se trabaja en áreas de enfermería que tratan de la asistencia hospitalaria, salud pública, enseñanza e investigación, así se percibe que la educación permanente en Bioseguridad, aunque es extremadamente importante para la prevención de riesgos respecto al trabajo porque puede generar compromiso de la salud del profesional o del cliente, tiende a ignorarse en muchos casos y esto aumenta los gastos con materiales, dias de ingreso, así como el compromiso del equipo de trabajo cuando hay accidentes que generan alejamiento del profesional de sus funciones. Esta observación e interés nos llevaron al curso de Especialización en Control de Infecciones en Asistencia a la Salud y a investigar la relación de la educación permanente y su efectividad en la reducción de costos ante los riesgos en el ambiente hospitalario. Pero, ¿qué es la Educación Permanente?

La Educación Permanente en Salud es una Política Nacional de Educación que según la Orden GM/Ms no 1996 de 20 de agosto de 2007, trata de la formación y desarrollo de trabajadores de SUS. Al instituirse, esta política propone crear acciones capaces de ayudar en el cambio de los procesos formativos y de las prácticas pedagógicas y de salud, englobando también la organización del trabajo. Ante esto, para que esta política genere resultados efectivos en las instituciones de salud es necesario que haya una labor articulada entre el sistema de salud, en sus esferas de gestión, y las 
instituciones formadoras, teniendo como objetivo la identificación de problemas rutinarios y la construcción de soluciones ${ }^{1}$.

La educación permanente es un proceso largo y continuo que transciende los límites educativos, se hace presente a lo largo de la vida de los individuos, existentes en una sociedad en continuos cambios ${ }^{23}$.

Los cambios tecnológicos y nuevos conocimientos movilizan las posibilidades y los saberes de los profesionales, llevándolos a la necesidad de continuidad en su formación².

La educación permanente debe lograr la incorporación de nuevas tecnologías ${ }^{3}$, de este modo, las labores de educación permanente en las instituciones de salud ofrecen oportunidades de aprendizaje como forma de educación en el trabajo posibilitándoles a los receptores de estas informaciones aprender de acuerdo con la necesidad y motivación².

En este contexto se incluye la Bioseguridad en la medida en que esta posibilita el análisis de los daños a que los profesionales de salud y de laboratorios se encuentran expuestos por sus actividades en el ambiente laboral.

Así, tras identificar los problemas derivados del ejercicio profesional que pueden resultar en riesgos es importante adoptar medidas de Bioseguridad.

La Bioseguridad se evidencia como un conjunto de medidas para prevenir, minimizar o eliminar riesgos provenientes de las actividades de investigación, producción, enseñanza, desarrollo tecnológico y prestación de servicios, que pueden comprometer la salud del hombre, de los animales, del medio ambiente o la calidad de los trabajos desarrollados.

Se resalta que las instituciones de salud y los trabajadores deben adoptar medidas de bioseguridad para minimizar exposiciones a situaciones que pueden resultar en daños ocupacionales ${ }^{4}$.

Los riesgos por los que los profesionales se exponen vienen de la jerarquización y complejidad de los hospitales o centro de salud, del tipo de atención hecho y del ambiente laboral. De esta forma, los profesionales de salud son más susceptibles de contraer enfermedades derivadas de accidentes de trabajo, por medio de procedimientos que presentan riesgos ${ }^{5}$. En esta perspectiva, la evaluación de los daños abarca varios aspectos como las buenas prácticas y la infraestructura de los laboratorios, agentes biológicos manipulados, así como la cualificación de los equipos ${ }^{6}$.

Basándose en estas afirmaciones, se entiende que adoptar medidas para evitar los daños requiere costos; por eso es necesario realizar el análisis costo-beneficio de las conductas llevadas a cabo por las instituciones de salud. Realizar el análisis costobeneficio permite estimar si el beneficio alcanzado en la intervención es justificable para el costo ${ }^{7}$.

De este modo, el estudio que se plantea se hace relevante al relacionar la Educación Permanente para la Bioseguridad como instrumento para la reducción de costos hospitalarios, al paso que asocia las actividades de prevención de accidentes a costoefectividad, contribuyendo a una mejor apreciación de las instituciones de salud. 
Ante lo expuesto, se presentan los siguientes objetivos: identificar si la bioseguridad puede contribuir a reducir el costo-efectividad en las hospitalizaciones, conocer el grado de impacto de la educación permanente para la bioseguridad en la reducción de los costos relacionados con la hospitalización y mostrar los efectos de la bioseguridad sobre la reducción de los costos hospitalarios basándonos en la literatura existente.

\section{METODOLOGÍA}

Se trata de una encuesta exploratoria descriptiva de abordaje cualitativo, de tipo revisión bibliográfica. Ésta busca proporcionar mayor familiaridad con el problema de estudio, haciéndolo más explícito ${ }^{8}$.

La encuesta descriptiva se caracteriza por la descripción de determinada población, fenómeno o relaciones entre variables, involucrando el uso de técnicas estándar de toma de datos ${ }^{9}$.

Este tipo de encuesta se preocupa de la realidad que no puede ser cuantificada, trabajando con el universo de significados, motivos, aspiraciones, creencias, valores y actitudes, lo que corresponde a un espacio más profundo de las relaciones, de los procesos y de los fenómenos que no pueden reducirse a la puesta en marcha de variables. Así, la metodología escogida proporciona amplía información y facilita la delimitación de la temática estudiada ${ }^{10}$.

De acuerdo con el procedimiento técnico/instrumento el tipo de estudio usado fue la encuesta de datos bibliográficos, que abarca toda la producción científica elaborada con respecto al tema, estudiado hasta hoy.

Al comienzo, se realizó la toma de datos sin recorte temporal de los artículos publicados en las bases de datos: BDENF (Banco de Datos de la Enfermería), LILACS (Literatura Latino-Americana y del Caribe en Ciencias de la Salud) y SCIELO (Scientific Electronic Library Online); para la discusión de los datos se seleccionaron solamente artículos de los últimos cinco años.

La búsqueda se realizó a través de los descriptores: análisis costo-beneficio; capacitación; educación en enfermería y exposiciones a agentes biológicos, además del refinamiento por el criterio de relevancia. Al término de la búsqueda, se analizaron los datos obtenidos a través del análisis de temáticas de contenido.

\section{RESULTADOS Y DISCUSIÓN}

La primera búsqueda a los descriptores se realizó de forma aislada y como resultado se obtuvieron diversas producciones, como muestra el cuadro uno (01). 
Cuadro 1 - Distribución cuantitativa de las bibliografías encontradas por descriptor en las bases de datos.

\begin{tabular}{|l|l|l|l|l|}
\hline \multirow{2}{*}{ Descriptores } & \multicolumn{3}{|l|}{ Base de Datos } & Total \\
\cline { 2 - 5 } & BDENF & LILACS & SCIELO & \\
\hline Análisis costo-beneficio & 11 & 705 & 3 & 719 \\
\hline Capacitación & 15 & 391 & 877 & 1283 \\
\hline Educación en Enfermería & 1715 & 2065 & 1366 & 5146 \\
\hline $\begin{array}{l}\text { Exposición a agentes } \\
\text { biológicos }\end{array}$ & 38 & 158 & 19 & 215 \\
\hline
\end{tabular}

Por el resultado presentado en el cuadro anterior se optó por refinar la investigación, y limitar mejor las producciones que contribuyen a responder los objetivos. Se realizó, entonces, una búsqueda con los descriptores asociados. Al realizar la asociación se adoptó como Operador Lógico Booleano el término "AND".

Cuadro 2 - Distribución cuantitativa de las bibliografías encontradas en las bases de datos, con los descriptores asociados en parejas.

\begin{tabular}{|l|l|l|l|l|}
\hline \multirow{2}{*}{ Descriptores } & \multicolumn{3}{|l|}{ Base de Datos } & Total \\
\cline { 2 - 5 } & BDENF & LILACS & SCIELO & \\
\hline Análisis costo-beneficio + Capacitación & 0 & 10 & 0 & 10 \\
\hline $\begin{array}{l}\text { Análisis costo-beneficio + Educación en } \\
\text { Enfermería }\end{array}$ & 0 & 1 & 0 & 1 \\
\hline $\begin{array}{l}\text { Análisis costo-beneficio + Exposición a } \\
\text { agentes biológicos }\end{array}$ & 0 & 0 & 0 & 0 \\
\hline Capacitación + Análisis costo-beneficio & 0 & 10 & 0 & 10 \\
\hline Capacitación + Educación en Enfermería & 170 & 956 & 0 & 1126 \\
\hline $\begin{array}{l}\text { Capacitación + Exposición a agentes } \\
\text { biológicos }\end{array}$ & 0 & 12 & 0 & 12 \\
\hline $\begin{array}{l}\text { Educación en Enfermería + Análisis } \\
\text { costo-beneficio }\end{array}$ & 0 & 1 & 0 & 1 \\
\hline Educación en Enfermería + Capacitación & 170 & 956 & 0 & 1126 \\
\hline $\begin{array}{l}\text { Educación en Enfermería + Exposición a } \\
\text { agentes biológicos }\end{array}$ & 7 & 9 & 0 & 16 \\
\hline $\begin{array}{l}\text { Exposición a agentes biológicos + } \\
\text { Análisis costo-beneficio }\end{array}$ & 0 & 0 & 0 & 0 \\
\hline $\begin{array}{l}\text { Exposición a agentes biológicos + } \\
\text { Capacitación }\end{array}$ & 0 & 12 & 0 & 12 \\
\hline $\begin{array}{l}\text { Exposición a agentes biológicos + } \\
\text { Educación en Enfermería }\end{array}$ & 7 & 9 & 0 & 16 \\
\hline
\end{tabular}

Al concluirse la búsqueda, se excluyó la base de Datos SCIELO porque no presenta resultados por medios de asociaciones y se realizó la lectura de los resúmenes de artículos que se encuentran completos en las demás bases con el objetivo de verificar el contenido de las obras. Esta conducta seleccionó bibliografías potenciales. 
Cuadro 3 - Selección de Bibliografías Potenciales.

\begin{tabular}{|c|c|c|c|c|}
\hline \multirow[t]{2}{*}{ Descriptores } & \multicolumn{2}{|c|}{ Base de Datos } & \multirow[t]{2}{*}{ Total } & \multirow{2}{*}{$\begin{array}{l}\text { Bibliografía } \\
\text { Potencial }\end{array}$} \\
\hline & BDENF & LILACS & & \\
\hline Análisis costo-beneficio + Capacitación & 0 & 10 & 10 & 0 \\
\hline $\begin{array}{l}\text { Análisis costo-beneficio + Educación en } \\
\text { Enfermería }\end{array}$ & 0 & 1 & 1 & 0 \\
\hline $\begin{array}{l}\text { Análisis costo-beneficio + Exposición a } \\
\text { agentes biológicos }\end{array}$ & 0 & 0 & 0 & 0 \\
\hline Capacitación + Análisis costo-beneficio & 0 & 10 & 10 & 0 \\
\hline $\begin{array}{l}\text { Capacitación } \\
\text { Enfermería }\end{array}$ & 170 & 956 & 1126 & 4 \\
\hline $\begin{array}{l}\text { Capacitación + Exposición a agentes } \\
\text { biológicos }\end{array}$ & 0 & 12 & 12 & 1 \\
\hline $\begin{array}{l}\text { Educación en Enfermería + Análisis } \\
\text { costo-beneficio }\end{array}$ & 0 & 1 & 1 & 0 \\
\hline $\begin{array}{llll}\begin{array}{l}\text { Educación } \\
\text { Capacitación }\end{array} & \text { en } & \text { Enfermería } & +\end{array}$ & 170 & 956 & 1126 & 3 \\
\hline $\begin{array}{l}\text { Educación en Enfermería + Exposición } \\
\text { a agentes biológicos }\end{array}$ & 7 & 9 & 16 & 5 \\
\hline $\begin{array}{l}\text { Exposición a agentes biológicos + } \\
\text { Análisis costo-beneficio }\end{array}$ & 0 & 0 & 0 & 0 \\
\hline $\begin{array}{l}\text { Exposición a agentes biológicos + } \\
\text { Capacitación }\end{array}$ & 0 & 12 & 12 & 1 \\
\hline $\begin{array}{l}\text { Exposición a agentes biológicos + } \\
\text { Educación en Enfermería }\end{array}$ & 7 & 9 & 16 & 5 \\
\hline
\end{tabular}

La selección de las bibliografías potenciales se basó en los artículos fundamentales que responden a los prerrequisitos de la encuesta. Posteriormente, se realizó la impresión y lectura de los artículos por completo con la finalidad de establecer relaciones textuales, contextuales e intertextuales, con vistas al surgimiento de categorías que permitan la discusión de los objetivos propuestos en el estudio en cuestión. En este momento, hubo la exclusión de artículos repetidos presentes en la misma categoría.

\section{Las contribuciones de la educación permanente para los enfermeros}

En esta categoría están insertas seis (6) producciones científicas, obsérvese el cuadro 4 que resalta las contribuciones de la educación permanente para los enfermeros.

Cuadro 4 - Distribución de las bibliografías potenciales de la categoría "Las contribuciones de la educación permanente para los enfermeros".

\begin{tabular}{|c|c|c|c|}
\hline Autor (es) & Año & Título & $\begin{array}{l}\text { Tipo de publicación/ } \\
\text { Fuente }\end{array}$ \\
\hline $\begin{array}{l}\text { JESUS, Maria Cristina } \\
\text { Pinto de; FIGUEIREDO, } \\
\text { Mariangela Aparecida } \\
\text { Gonçalves; SANTOS, } \\
\text { Sueli Maria dos Reis; } \\
\text { AMARAL, Arlete M}{ }^{\underline{a}}\end{array}$ & 2011 & $\begin{array}{l}\text { Educación permanente } \\
\text { en enfermería en un } \\
\text { hospital universitario. }\end{array}$ & $\begin{array}{l}\text { Artigo / Rev. Esc Enferm } \\
\text { USP; } 45 \quad \text { (5): } 1229 \text { - } \\
\begin{array}{ll}\text { 1236, out. } & \end{array}\end{array}$ \\
\hline
\end{tabular}




\begin{tabular}{|c|c|c|c|}
\hline $\begin{array}{lrr}\text { Moreira do; } & \text { ROCHA, } \\
\text { Letícia de } & \text { Oliveira; } \\
\text { THIOLLENT, } & \text { Michel } \\
\text { Jean Marie. } & \\
& \end{array}$ & & & \\
\hline 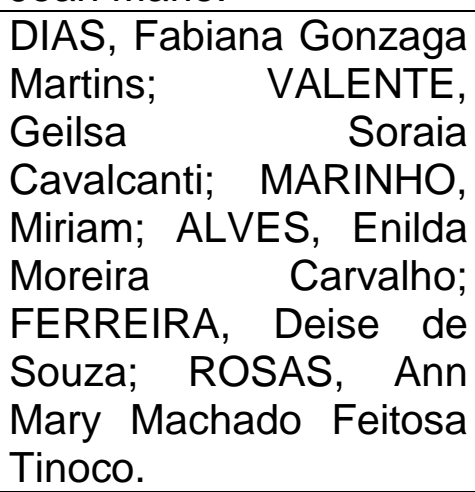 & $\begin{array}{r}2010 \\
\\
\end{array}$ & $\begin{array}{l}\text { La } r \text { educación } \\
\text { permanente en un } \\
\text { equipo de enfermería } \\
\text { para prevenir la } \\
\text { infección hospitalaria. }\end{array}$ & $\begin{array}{l}\text { Artigo / Rev. Enferm. } \\
\text { UFPE on line; } 4 \text { (1): } 327 \\
\text { - 335, 20100300. }\end{array}$ \\
\hline $\begin{array}{l}\text { MONTANHA, Dionize; } \\
\text { PEDUZZI, Marina. }\end{array}$ & 2010 & $\begin{array}{l}\text { Educación permanente } \\
\text { en enfermería; } \\
\text { levantamiento de } \\
\text { necesidades y } \\
\text { resultados esperados } \\
\text { según la concepción } \\
\text { de los trabajadores. }\end{array}$ & $\begin{array}{llr}\text { Artigo / Rev. } & \text { Esc. } \\
\text { Enferm. } & \\
\text { USP; } 44 & \text { (3): } 597 & \text { - 604, } \\
\text { set. }\end{array}$ \\
\hline $\begin{array}{l}\text { PALHARES } \text { Guimarães, } \\
\text { Eliane } \\
\text { HAUEISEN Marina; } \\
\text { Sandra; PAOLINELLI } \\
\text { Rabelo, Flávia Cristina. }\end{array}$ & 2010 & $\begin{array}{l}\text { Educación permanente } \\
\text { en salud: reflexiones y } \\
\text { desafíos. }\end{array}$ & $\begin{array}{l}\text { Artigo / Cienc. Enferm; } \\
16 \text { (2): } 25 \text { - 33, ago. }\end{array}$ \\
\hline $\begin{array}{lr}\text { SILVA, Luiz } \text { Anildo } \\
\text { Anacleto da; FERRAZ, } \\
\text { Fabiane; LINO, Mônica } \\
\text { Motta; BACKES, Vânia } \\
\text { Marli } \\
\begin{array}{lr}\text { SCHMIDT, Schubert; } \\
\text { Marcia Soares. Sandra }\end{array} \\
\end{array}$ & 2010 & $\begin{array}{l}\text { Educación permanente } \\
\text { en salud y en el trabajo } \\
\text { de enfermería: } \\
\text { perspectiva de una } \\
\text { praxis transformadora. }\end{array}$ & $\begin{array}{l}\text { Artigo / Rev. } \text { Gaúch. } \\
\text { Enferm; } 31 \quad \text { (3): } 557 \text { - } \\
\text { 561, set. }\end{array}$ \\
\hline $\begin{array}{l}\text { PASCHOAL, Amarílis } \\
\text { Schiavon; MANTOVANI, } \\
\text { Ma de Fátima; MÉIER, }^{\text {a }} \text { Marineli Joaquim. }\end{array}$ & 2007 & $\begin{array}{lrr}\text { Percepción } & \text { de } & \text { la } \\
\text { educación permanente, } \\
\text { continuada y ran } \\
\text { servicio } & \text { para } \\
\text { enfermeros de } & \text { un } \\
\text { hospital de enseñanza. }\end{array}$ & $\begin{array}{l}\text { Artigo / Rev. Esc. } \\
\text { Enferm. USP; } 41 \text { (3): } 478 \\
\text { - 484, set. }\end{array}$ \\
\hline
\end{tabular}

La educación es una herramienta indispensable y primordial si consideramos los constantes cambios derivados de la globalización. En este contexto se obtienen nuevos saberes y prácticas en todo momento con prioridad a la calidad y excelencia. Así, la educación es el proceso utilizado para procurar a los sujetos el conocimiento, experiencias culturales, científicas, y adaptativas necesarias a su actuación en el medio social ${ }^{11}$.

Los profesionales de salud también deben ser insertados en un proceso de educación, siendo este de cuño profesional, donde la educación permanente se encuentra integrada. Y para el desarrollo de este modelo educacional el enfermero es primordial $^{12}$. 
La educación permanente contribuye a la práctica profesional y esto se evidencia cuando los profesionales se hacen cargo al ejercer el cuidado, a través del compromiso auto firmado en el momento en que se motiva la búsqueda de autoconocimiento, perfeccionamiento y actualización ${ }^{13}$.

Al ejercer este compromiso se propone mejorar el cuidado que es prestado al cliente y a la comunidad y al mismo tiempo ocurren procesos de cambios y cualificación en la conducta del profesional de enfermería, lo que resulta en calidad en la asistencia.

En este sentido, una praxis transformadora proporciona a los profesionales la construcción de conocimientos teniendo como base la libertad tanto individual como colectiva en que estos conocimientos encuentran ancla en los preceptos de ciudadanía $^{14}$, o sea, aunque la transformación del profesional por medio de la enseñanza ocurra de manera individual, esta conducta incide en cambios sociales, llevando a la práctica profesional competente, consciente y responsable, además promueve en los profesionales una conciencia sobre responsabilidad auto educacional $^{13}$.

De este modo, se verifica que la educación permanente es una herramienta importante para identificar las necesidades de los profesionales de salud, pues permite explotar las necesidades educativas partiendo del punto de vista de los trabajadores ${ }^{15}$ y objetiva organizar el trabajo de Enfermería en articulación con las prácticas de la profesión en los diversos sectores hospitalarios ${ }^{12,16}$, sin embargo, aunque la educación permanente sea positiva para el ejercicio profesional, es imprescindible tratar de una cuestión, es decir, el hecho de que los profesionales de enfermería aún presentan una falsa idea de lo que es la educación permanente ${ }^{11,16}$.

Estudios señalan que enfermeros presentan dificultades a la hora de diferenciar qué es educación permanente, pero cuando se discute la terminología de la palabra estos consiguen entender la diferencia que hay entre educación permanente, educación continua y en trabajo ${ }^{11}$. Otro estudio muestra que cuando les cuestionan, los profesionales presentan predominantemente en sus hablas características de la educación continua; los profesionales tienden a generar la unión entre los tipos de educación cuando resaltan que las necesidades educacionales deben estar dirigidas a las fallas identificadas en el proceso de trabajo, para los problemas más frecuentes y conductas que busquen la amplia autonomía profesional ${ }^{16}$. Sin embargo, se observó que la educación continua puede sumarse a la educación permanente, al estimular en los profesionales la conciencia de que son responsables por el propio proceso permanente de capacitación ${ }^{12}$.

\section{La Educación permanente frente al riesgo biológico}

En esta categoría están insertas seis (6) producciones científicas, ved cuadro 5, que resalta las contribuciones de la educación permanente frente al riesgo biológico. 
Cuadro 5 - Distribución de las bibliografías potenciales de la categoría "La Educación permanente frente al riesgo biológico".

\begin{tabular}{|c|c|c|c|}
\hline Autor (es) & Año & Título & $\begin{array}{l}\text { Tipo de publicación/ } \\
\text { Fuente }\end{array}$ \\
\hline $\begin{array}{l}\text { CANALLI, Rafaela Thaís } \\
\text { Colombo; } \quad \text { MORIYA, } \\
\text { Tokico } \quad \text { Murakawa; } \\
\text { HAYASHIDA, Miyeko. }\end{array}$ & 2011 & $\begin{array}{lr}\text { Prevención } & \text { de } \\
\text { accidentes } & \text { con } \\
\text { material biológico entre } \\
\text { estudiantes } \\
\text { enfermería. }\end{array}$ & $\begin{array}{l}\text { Artigo / Rev. enferm. } \\
\text { UERJ; } 19 \text { (1): } 100-106 \text {, } \\
\text { jan. - mar. }\end{array}$ \\
\hline $\begin{array}{lr}\text { GUILARDE, } & \text { Adriana } \\
\text { Oliveira; OLIVEIRA, Ana } \\
\text { Ma de; } & \text { TASSARA, } \\
\text { Marianna; } & \text { OLIVEIRA, } \\
\text { Bethânia } & \text { de; } \\
\text { ANDRADE, } & \text { Sabrina } \\
\text { Sgambatti de. } & \end{array}$ & 2010 & $\begin{array}{l}\text { Accidentes con } \\
\text { material biológico entre } \\
\text { profesionales de } \\
\text { Hospital Universitario } \\
\text { en Goiânia. }\end{array}$ & $\begin{array}{l}\text { Rev. Patol. Trop; } 39 \text { (2): } \\
131 \text { - 136, abri. - jun. }\end{array}$ \\
\hline $\begin{array}{l}\text { NEVES, Heliny Carneiro } \\
\text { Cunha; } \\
\text { Adenícia Custódia Silva } \\
\text { e; BARBOSA, Jackeline } \\
\text { Maciel; RIBEIRO, Luana } \\
\text { Cássia r Miranda; } \\
\text { TRIPPLE, Anaclara } \\
\text { Ferreira Veiga; ALVES, } \\
\text { Sergiane } \\
\text { SUZUKI, Karina. }\end{array}$ & 2010 & $\begin{array}{ll}\text { El uso } & \text { de } \\
\text { equipamientos } & \text { de } \\
\text { protección individual } \\
\text { por profesionales en } \\
\text { unidades } \\
\text { endoscopia. }\end{array}$ & $\begin{array}{l}\text { Artigo / Rev. Enferm. } \\
\text { UERJ; } 18 \text { (1): } 61-66 \text {, } \\
\text { jan. - mar. }\end{array}$ \\
\hline $\begin{array}{l}\text { ALMEIDA, André Nunes } \\
\text { Gomes de; TRIPPLE, } \\
\text { Anaclara Ferreira Veiga; } \\
\text { SOUZA, Adenícia } \\
\text { Custodio Silva e; } \\
\text { BRASILEIRO, Marislei } \\
\text { Espíndula. }\end{array}$ & 2009 & $\begin{array}{l}\text { Riesgo biológico entre } \\
\text { los trabajadores de } \\
\text { enfermería. }\end{array}$ & $\begin{array}{l}\text { Artigo / Rev. Enferm. } \\
\text { UERJ; } 17 \text { (4): } 595-600 \text {, } \\
\text { out. - dez. }\end{array}$ \\
\hline $\begin{array}{lcr}\text { BONINI, } & \text { Aline } & M^{\mathrm{a}} \text {; } \\
\text { ZEVIANI, Camila } & \text { de } \\
\text { Paula; FACCHIN, Luiza } \\
\text { Tayar; GIR, } & \text { Elucir; } \\
\text { CANINI, Silvia } & \text { Rita } \\
\text { Marin da Silva. }\end{array}$ & 2009 & $\begin{array}{l}\text { Exposición } \\
\text { ocupacional de los } \\
\text { profesionales } \\
\text { enfermería de una } \\
\begin{array}{l}\text { unidad de terapia } \\
\text { intensiva a material } \\
\text { biológico. }\end{array}\end{array}$ & $\begin{array}{l}\text { Rev. Electrônica enferm. } \\
\text {; } 11 \text { (3) set. }\end{array}$ \\
\hline $\begin{array}{l}\text { LOUREIRO, Lívia Agy; } \\
\text { GOMES, Ana Carolina; } \\
\text { MALAGUTI, Silmara } \\
\text { Elaine; CANINI, Silvia } \\
\text { Rita Marin da Silva; } \\
\text { MACHADO, Alcyone } \\
\text { Artioli; GIR, Elucir. }\end{array}$ & 2009 & $\begin{array}{lr}\text { Adhesión } & \text { de } \\
\text { profesionales } & \text { de } \\
\text { enfermería } & \text { al } \\
\text { seguimiento } & \text { clínico } \\
\text { tras } & \text { exposición } \\
\text { ocupacional } & \text { con } \\
\text { material biológico. } & \end{array}$ & $\begin{array}{l}\text { Rev. Electrônica enferm.; } \\
11 \text { (2), jun. }\end{array}$ \\
\hline
\end{tabular}

Los riesgos están presentes en todas las áreas de una institución donde haya contacto con el paciente y/ o sus residuos biológicos ${ }^{17}$. Por eso, al desarrollarse 
actividades que promueven contacto con sangre y fluídos corporales, los profesionales de salud están expuestos a lo que se denomina Riesgo biológico.

El Riesgo biológico consiste en la posibilidad de contacto con materiales biológicos que pueden generar daños a la salud por ser vehículos de agentes biológicos patogénos $^{18}$. De este modo, es necesario adoptar medidas que busquen prevenir, minimizar o eliminar los riesgos mediante la adopción de normas de bioseguridad y precauciones estándar ${ }^{18}$. $\mathrm{Y}$ estas medidas son indispensables por parte de los profesionales al cuidar al paciente, independientemente de las condiciones de salud del mismo.

Los profesionales de salud se encuentran expuestos a riesgos biológicos y químicos ${ }^{19}$ y esta exposición se debe a los principales factores: ausencia de equipos de protección individual en el sector ${ }^{18}$, su no utilización por los profesionales ${ }^{18}$, como $^{2}$ también su uso indebido ${ }^{19}$.

Entre las profesiones más susceptibles se encuentra la enfermería y esto puede relacionarse con la mayor cantidad de estos profesionales en la labor de salud, así como una asistencia que necesita de mayor contacto con el paciente, tipo y frecuencia de los procedimientos ${ }^{17}$.

La exposición ocupacional promueve en los profesionales de salud consecuencias derivadas de la exposición a la sangre y otros fluidos corpóreos, pero eso no se refiere sólo a las infecciones aunque esta sea una de las que da más recelo ${ }^{18,20}$. Ésta genera también traumas psicológicos derivados de la posibilidad de una seroconversión, efectos de las drogas profilácticas, cambios de relaciones y prácticas sexuales $^{18}$.

La educación de los profesionales de salud debe partir del mecanismo de ocurrencia de accidentes englobando material biológico y la evaluación del proceso de trabajo, estableciéndose medidas que resulten en un ambiente de prácticas seguras ${ }^{21}$, sin embargo al plantear con los profesionales los accidentes con materiales biológicos no se debe enfatizar solamente la adopción de medidas preventivas para reducción del número de accidentes, sino la importancia de un esquema vacunal completo y evaluación de los títulos de Anti - HBs después la vacunación ${ }^{20}$. Por eso, es necesario programa de educación permanente, para desarrollar en los profesionales competencias de carácter cognitivo, psicomotor y actitudinales ${ }^{19}$.

El programa de educación permanente debe también buscar la adhesión de los profesionales de enfermería al seguimiento clínico recomendado en caso de exposición a materiales biológicos en respuesta a dificultades de adhesión a las medicinas para prevención del transmisor del VIH tras la exposición ocupacional ${ }^{22}$. Así, es urgente el compromiso de los profesionales educadores.

\section{Las contribuciones de la educación permanente para la Bioseguridad}

En esta categoría se seleccionó una (1) producción científica, veáse cuadro 6 (seis), que resalta las contribuciones de la educación permanente para la bioseguridad. 
Cuadro 6 - Distribución de las bibliografías potenciales de la categoría "Las contribuciones de la educación permanente para la bioseguridad".

\begin{tabular}{|c|c|c|c|}
\hline Autor (es) & Año & Título & $\begin{array}{l}\text { Tipo de publicación/ } \\
\text { Fuente }\end{array}$ \\
\hline $\begin{array}{lrr}\text { BONINI, } & \text { Aline } & \text { Má; } \\
\text { ZEVIANI, Camila } & \text { de } \\
\text { Paula; FACCHIN, Luiza } \\
\text { Tayar; GIR, } & \text { Elucir; } \\
\text { CANINI, Silvia } & \text { Rita } \\
\text { Marin da Silva. } & \end{array}$ & 2009 & $\begin{array}{l}\text { Exposición } \\
\text { ocupacional de los } \\
\text { profesionales } \\
\text { enfermería de una } \\
\begin{array}{l}\text { unidad de } \\
\text { intensiva a } \\
\text { biológico. }\end{array}\end{array}$ & $\begin{array}{l}\text { Artigo / Rev. Electrônica } \\
\text { enferm; 11(3), set. }\end{array}$ \\
\hline
\end{tabular}

La Bioseguridad es un conjunto de medidas que deben ser adoptadas por las instituciones de salud y por los trabajadores para minimizar exposiciones a situaciones que pueden resultar en riesgos ocupacionales ${ }^{2}$, pero pese al conocimiento sobre los riesgos, medidas de protección y seguridad, éstas, a menudo, son dejadas de lado lo que genera exposición a los riesgos existentes y hasta incluso enfermedades ocupacionales ${ }^{23}$.

Asociar la educación permanente con bioseguridad es extremadamente relevante para adoptar medidas preventivas teniendo como base los riesgos y las causas de los accidentes a los que los profesionales están sujetos ${ }^{24}$, pues a pesar de que los profesionales conocen los riesgos a los que están expuestos, esto no garantiza la práctica de comportamientos seguros en el trabajo. De este modo, la educación permanente dirigida a la bioseguridad proporciona condiciones para desarrollar prácticas profesionales seguras desde que promueva discusiones crítico reflexivas de los profesionales sobre su praxis.

El estudio de los autores seleccionados para esta categoría evidencia que pese al conocimiento obtenido por medio de cursos / conferencias sobre los riesgos a que están expuestos y de la necesidad del uso de equipos de protección individual (EPIs), muchos profesionales en situaciones diversas no usan los EPls lo que según los autores hace necesaria la revisión de las estrategias ${ }^{21}$. Ante lo expuesto, resaltamos que la educación permanente debe realizarse de manera continua y con la metodología adoptada para su desarrollo centrada en la resolución de problemas con vistas a cambios efectivos ${ }^{12}$. Así, la bioseguridad entendida como base educacional, es además de fundamental, estratégica desde el punto de vista científico, pues garantiza tanto la bioseguridad de los profesionales como del ambiente ${ }^{25}$.

Luego, programas de educación permanente promueven contribuciones y consecuentemente concienciación de los profesionales de enfermería sobre la seguridad necesaria al ejercicio profesional de modo más efectivo cuando comparado con la capacitación y cursos esporádicos ${ }^{21}$.

\section{Las contribuciones del análisis costo- beneficio para la salud}

La búsqueda por descriptores asociados a "Análisis costo - beneficio" para la realización de esta investigación resultó en referencias bibliográficas limitadas (ver cuadro 2), y al seleccionar entre estas bibliografías como base para la discusión se evidencia la existencia de artículos a utilizare (ver cuadro 3), siendo necesario recurrir 
a la selección realizada con descriptores de manera individualizada, así se evidencia la gran relevancia en la temática discutida.

En esta selección se observó que la mayoría de los artículos con la temática análisis costo - beneficio están relacionados con patologías o trasplantes, o sea, consideran solamente las conductas médicas sobre el paciente, pero debemos reflexionar y comprender que en torno a estas conductas se encuentran medidas de bioseguridad que utilizan cada vez más tecnologías contra los riesgos biológicos.

En esta categoría están insertas cuatro (4) producciones científicas, ver cuadro 7 (siete), que resalta las contribuciones del análisis costo - beneficio para la salud.

Cuadro 7 - Distribución de las bibliografías utilizadas en la categoría "Las distribuciones del análisis costo - beneficio para la salud".

\begin{tabular}{|c|c|c|c|}
\hline Autor (es) & Año & Título & $\begin{array}{l}\text { Tipo de publicación/ } \\
\text { Fuente }\end{array}$ \\
\hline $\begin{array}{l}\text { RIBEIRO, José } \\
\text { Mendes; SIQUEIRA, } \\
\text { Sandra Aparecida } \\
\text { Venâncio de; PINTO, } \\
\text { Luiz Felipe de Silva. }\end{array}$ & 2010 & $\begin{array}{l}\text { Evaluación de la } \\
\text { atención a la salud de } \\
\text { los niños (0-5 años) en } \\
\text { el PSF de Teresópolis } \\
\text { (RJ) según la } \\
\text { percepción de los } \\
\text { usuarios. }\end{array}$ & $\begin{array}{l}\text { Artigo / Ciênc. Saúde } \\
\text { Coletiva; } 15 \text { (2): } 517 \text { - } \\
527 .\end{array}$ \\
\hline $\begin{array}{l}\text { SECOLI, Silvia Regina; } \\
\text { NITA, Marcelo Eidi; } \\
\text { ONO-RITA, Suzane } \\
\text { Kioko; NOBRE, Moacyr }\end{array}$ & 2010 & $\begin{array}{l}\text { Evaluación de } \\
\text { tecnología en salud: II. } \\
\text { Análisis de costo- } \\
\text { efectividad. }\end{array}$ & $\begin{array}{l}\text { Artigo / } \quad \text { Arq. } \\
\text { Gastroenterol; } 47 \text { (4): } 329 \\
-333 .\end{array}$ \\
\hline $\begin{array}{l}\text { NITA, Marcelo Eidi; } \\
\text { SECOLI, Silvia Regina; } \\
\text { NOBRE, Moacyr; ONO- } \\
\text { NITA, Suzane Kioko. }\end{array}$ & 2009 & $\begin{array}{ll}\text { Métodos } & \text { de } \\
\text { investigación } & \text { en } \\
\text { evaluación } & \text { de } \\
\text { tecnología en salud. }\end{array}$ & $\begin{array}{l}\text { Artigo / } \quad \text { Arq. } \\
\text { Gastroenterol; } 46 \text { (4): } 252 \\
-255 .\end{array}$ \\
\hline $\begin{array}{ll}\text { MELO, Juliana } & \text { Carrijo; } \\
\text { NETO, } & \text { Miguel } \\
\text { Cendoroglo. } & \end{array}$ & 2008 & $\begin{array}{lr}\text { Evaluación } & \text { de } \\
\text { tecnología de salud } \\
\text { (ATS) y } \\
\text { implicaciones en la } \\
\text { práctica clínica. }\end{array}$ & 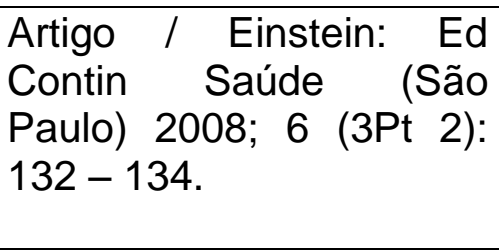 \\
\hline
\end{tabular}

Debido a la creciente incorporación de nuevas tecnologías incluso las que se refieren a la salud - medicina, procedimientos médicos, equipos y programas de cuidados para la salud - solo aquellas con costo y efectividad comprobada, tienden a ser adoptadas en los sistemas de salud y hospitales, siendo este público o privado ${ }^{26,27}$. Así, la evaluación económica, análisis costo- beneficio es utilizada como instrumento de ayuda al elegir nuevas tecnologías en salud, aunque pueda existir aspectos de orden epidemiológico y cultural interviniendo en esta decisión ${ }^{27}$. Pero, ¿qué es análisis costo-beneficio?

Es una forma de evaluación donde los resultados referentes a las tecnologías / programas son valorados en unidades monetarias. El objetivo de este análisis es servir de indicador de efectividad, promoviendo criterios de decisión que se basen en las alternativas que aceptan menores costos y mayores beneficios, siendo uno de los principales métodos de evaluación económica en el área de salud. 
Innovaciones tecnológicas son estimulantes para la oferta de nuevos servicios, pero entrelazado con eso están los riesgos de la adopción de una determinada técnica o toma de decisión, en la cual los límites de efectividad pueden comprometer la eficiencia del tratamiento hecho en detrimento de límites de efectividad próximos o no comprobados $^{28}$.

Es importante realizar la cuantificación de las necesidades y valoración de los tratamientos recurriendo a una metodología que enfoque los métodos del capital humano, de las decisiones sociales y del valor del riesgo previsto $^{28}$.

Por lo tanto, evaluar el costo - beneficio de acciones y servicios de salud es primordial para elaborar programas y estrategias que respondan a las reales necesidades de la población, así como justificar estrategias y programas a adoptar ${ }^{29}$.

\section{CONCLUSIÓN}

Se concluye que la Educación Permanente contribuye a fortalecer las prácticas de los profesionales de enfermería, teniendo en cuenta que este tipo de educación promueve en los profesionales la conciencia de que son sujetos en su proceso permanente de capacitación.

En cuanto a asociar la Educación Permanente con la Bioseguridad, percibimos que esta genera la adopción de prácticas profesionales seguras; oriundas de la concienciación de los profesionales. Además, percibimos que a partir de esta concienciación ocurre la adopción de comportamientos seguros en el trabajo, luego la Bioseguridad vista aisladamente como un conjunto de medidas que buscan la protección contra los riesgos inherentes a las actividades desarrolladas profesionalmente es de gran eficacia, pero cuando estas medidas son desarrolladas junto a la educación permanente, las conductas de prevención de los riesgos son más duraderas y consecuentemente más eficientes.

A lo largo de este estudio se evidenció la existencia de artículos que abordan los efectos de la educación permanente así como de la Bioseguridad sobre el costobeneficio en las hospitalizaciones, pero pese a las dificultades encontradas para basar el estudio fue posible constatar que la Educación Permanente unida a Bioseguridad tiende a promover la reducción de los costos relacionados con la hospitalización, a partir del momento que medidas que intentan prevenir, minimizar o eliminar la exposición a los riesgos son adoptadas por las instituciones de salud y por los trabajadores. Estas medidas resultan en la reducción o eliminación de los posibles agentes que tienden a comprometer la salud del profesional o cliente y consecuentemente promueven la reducción de gastos de materiales, días de ingreso, así como evita el compromiso del equipo en detrimento de una exposición ocupacional que puede generar alejamiento del profesional descabalando al equipo.

Es importante resaltar el desarrollo de nuevas investigaciones que aborden la temática estudiada proporcionando mayor acervo a los investigadores, profesionales y estudiantes del área de salud. 


\section{REFERENCIAS}

1. Ministério da Saúde (Brasil). Portaria $n^{\circ}$. 1.996, de 20 de agosto de 2007. Dispõe sobre as diretrizes para a implementação da Política Nacional de Educação Permanente em Saúde. Diário Oficial da União 22 ago 2007.

2. Monteiro MI; Chillida MSP; Bargas EB.Educação continuada em um serviço terceirizado de limpeza de um hospital universitária. Rev. Latino-Am. Enfermagem. 2004; 12(3): 6-12.

3. Ricaldoni CAC; Sena RR de. Educação permanente: uma ferramenta para pensar e agir no trabalho de enfermagem. Rev. Latino-Am. Enfermagem. 2006; 14(6): 837-842. 4. Soares LG; Labronici LM; Maftum MA; Sarquis LMM; Kirchhof AL. Risco Biológico em trabalhadores de Enfermagem: Promovendo a Reflexão e a prevenção. Cogitare Enferm. 2011; 16(2): 261-7.

5. Carvalho CMRS; Madeira MZ de A; Tapety FI; Alves ELM; Martins M do C de C; Brito JNP de O. Aspectos de biossegurança relacionados ao uso do jaleco pelos profissionais de saúde: uma revisão da literatura. Texto contexto - enferm. 2009; 18(2): 355-360.

6. Ministério da Saúde (Brasil). Diretrizes gerais para o trabalho em contenção com Agentes Biológicos. Brasília: Editora MS, 2006b.

7. Vianna D. Há relação entre custo-efetividade de acordo com diferentes metas?. Rev. Bras.Hipertens.2010. Vol.17(3): 182-185.

8. Figueiredo, NMA de. Método e Metodologia na pesquisa científica. $2^{\underline{a}}$ ed. rev. São Caetano do Sul, SP: Yendis, 2007.237 p.

9. Silva EL da. Metodologia da pesquisa e elaboração de dissertação/Edna Lúcia da Silva, Estera Muszkat Menezes. - 3.ed. Rev. Atual. - Florianópolis: Laboratório de Ensino a Distância da UFSC, 2001. Disponível em www.ucs.br/ccet/defg/vvbgmiss/aula1.pdf acesso em: 01 de nov de 2011.

10. Minayo, MCS. (Org.). Pesquisa social: teoria, método e criatividade. Rio de Janeiro: Vozes, 2002. 80 p.

11. Paschoal AS; Mantovani MF; Méier MJ. Percepção da educação permanente, continuada e em serviço para enfermeiros de um hospital de ensino. Rev esc enferm USP [periódico on line]. 2007 Set [citado 15 dez 2011]; 41(3):478-484. Disponível em: http://www.scielo.br/scielo.php?script=sci arttext\&pid=S008062342007000300019\&ln

$\mathrm{g}=\mathrm{en}$.

12. Dias FGM; Valente GSC; Chrizostimo MM; Alves EMC; Ferreira D de S; Rosas AMMFT. The permanent education in the nursing team to prevent the hospital infection. Rev enferm UFPE on line [periódico on line]. 2010 jan / mar.[citado $29 \mathrm{dez}$ 2011]; $\quad 4 \quad(1): 324-32 . \quad$ Disponível $\quad$ em: http://www.ufpe.br/revistaenfermagem/index.php/revista/article/view/684/pdf 326.

13. Jesus MCP de; Figueiredo MAG; Santos SM dos R; Amaral AMM do; Rocha $L$ de $\mathrm{O}$; Thiollent MJM. Educação permanente em enfermagem em um hospital universitário. Rev. esc. enferm. USP [periódico on line]. 2011 Out [citado $30 \mathrm{dez}$ 2011] ; 45(5): 1229-1236. Disponível em: http://www.scielo.br/scielo.php?script=sci arttext\&pid=S008062342011000500028\&ln $\mathrm{g}=\mathrm{pt}$.

14. Silva LAA da; Ferraz F; Lino MM; Backes VMS; Schmidt SMS. Educação permanente em saúde e no trabalho de enfermagem: perspectiva de uma práxis transformadora. Rev. Gaúcha Enferm. (Online) [periódico on line]. 2010 Set [citado 14 dez 2011] ; 31(3): 557-561. Disponível em: http://www.scielo.br/scielo.php?script=sci arttext\&pid $=\$ 198314472010000300021 \&$ ln $\mathrm{g}=\mathrm{en}$. 
15. Palhares GEM; Haueisen MS; Paolinelli RFC. EDUCAÇÃO PERMANENTE EM SAÚDE: Reflexões e desafios. Cienc. enferm. [periódico on line]. 2010 Ago [citado 15 Dez 2012]; 16(2): 25-33. Disponível em: http://www.scielo.cl/scielo. php?script=sci arttext\&pid=S071795532010000200004\&lng=es.

16. Montanha D; Peduzzi M. Educação permanente em enfermagem: levantamento de necessidades e resultados esperados segundo a concepção dos trabalhadores. Rev. esc. enferm. USP [periódico na Internet]. 2010 Set [acesso em 2011 Dez 15] ; 44(3): 597-604. Disponível em: http://www.scielo.br/scielo.php?script=sci arttext\&pid=S008062342010000300007\&ln $\mathrm{g}=\mathrm{pt}$.

17. Almeida ANG de; Tipple AFV; Souza ACS e; Brasileiro, ME. Risco biológico entre os trabalhadores de enfermagem. Rev. Enferm. UERJ. 2009; 17 (4): 595-600.

18. Canalli RTC; Moriya TM; Hayashida M. Prevenção de acidentes com material biológico entre estudantes de enfermagem. Rev. Enferm. UERJ. 2011; 19 (1): 100106.

19. Neves HCC; Souza ACS e; Barbosa JM; Ribeiro LCM; Tipple AFV; Alves SB; Suzuki K. O uso de equipamentos de proteção individual por profissionais em unidades de endoscopia. Rev. Enferm. UERJ. 2010; 18 (1): 61-66.

20. Guilarde AO; Oliveira AM de; Tassara M; Oliveira B de; Andrade, SS de. Acidentes com material biológico entre profissionais de Hospital Universitário em Goiânia. Rev. Patol. Trop. 2010; 39 (2): 131-136.

21. Bonini AM; Zeviani CP; Canini SRMS. Exposição ocupacional dos profissionais de enfermagem de uma unidade de terapia intensiva a material biológico. Rev. Eletr. Enf. [periódico on line]. 2009 Set. [citado13 dez 2011]; 11(3):658-664. Disponível em: http://www.fen.ufg.br/revista/v11/n3/v11n3a25.htm.

22. Loureiro LA; Gomes AC; Malaguti SE; Canini SEM da S; Machado AA; Gir E.Adesão de profissionais de enfermagem ao seguimento clínico após exposição ocupacional com material biológico. Rev. Eletr. Enf. [periódico on line]. 2009 Jun [citado 13 dez 2011]; 11(2): 303-308. Disponível em: http://www.fen.ufg.br/revista/v11/n2/v11n2a10.htm.

23. Castro MRC; Faria SNP. Repercussions of accident with perforating-cutting instruments for the nursing: a construction based on the focal group. Esc Anna Nery Rev Enferm.2009. 13(3):523-29.

24. Silva MR; Cortez EA; Valente GSC. Accident with materials piercing and biological environment in the hospital: analysis of risk exposure and preventive measures. R.pesq.: cuid. fundam. online.[periódico on line] $2011 \mathrm{abr} / j u n$;[citado 10 dez 2011]; 3(2): 1856-72. Disponível em: http://www.seer.unirio.br/index.php/cuidadofundamental/article/viewArticle/1280 25. Silva ADRI da; Mastroeni MF. Biossegurança: o conhecimento dos formandos da área da saúde. Rev.baiana saúde pública. 2009; 33(4): 654-665.

26. Nita ME; Secoli SR; Nobre M; Ono-Nita SK. Métodos de pesquisa em avaliação de tecnologia em saúde. Arq. Gastroenterol. [periódico on line]. 2009 Dez [citado 06 Jan 2012]; 46(4): 252-255. Disponível em: http://www.scielo.br/scielo.php?script=sci arttext\&pid=S000428032009000400002\&ln $\mathrm{g}=\mathrm{en}$.

27. Secoli SR; Nita ME; Ono-Nita SK; Nobre M. Avaliação de tecnologia em saúde: II. A análise de custo-efetividade. Arq. Gastroenterol. [periódico on line]. 2010 Dez [citado 06 Jan 2012]; 47(4): 329-333. Disponível em: http://www.scielo.br/scielo.php?script=sci arttext\&pid=S000428032010000400002\&ln $\mathrm{g}=\mathrm{en}$.

28. Melo JC; Neto MC. Avaliação de tecnológica de saúde (ATS) e suas implicações na prática clínica. Einstein: Ed Contin Saúde (São Paulo) 2008; 6 (3 Pt 2): 132-134. 
29. Ribeiro JM; Siqueira SAV; Pinto LFS. Avaliação da atenção à saúde da criança (05 anos) no PSF de Teresópolis (RJ) segundo a percepção dos usuários. Ciênc. Saúde Coletiva. 2010. 15(2): 517-527. 